\section{Market Withdrawal of Vioxx: Is it Time to Rethink the Use of COX-2 Inhibitors?}

On September 30, 2004, Merck \& Co. Inc., the manufacturer of the arthritis drug rofecoxib (Vioxx), announced the worldwide voluntary market withdrawal of rofecoxib after a study confirmed long-standing concerns that use of the drug raises the risk of heart attack and stroke. ${ }^{1}$ The drug was aggressively marketed after receiving U.S. Food and Drug Administration (FDA) approval in 1999 and has resulted in more than 84 million prescriptions worldwide. More than 2 million people were taking Vioxx at the time of the recall, making this the largest voluntary drug recall in history. ${ }^{2}$

The recall has substantial financial implications as well. Vioxx brought in more than $\$ 2.5$ billion annually for Merck and was responsible for approximately $10 \%$ of its worldwide sales. On the day of the announcement, the value of Merck's stock dropped by approximately $\$ 25$ billion. In addition, litigationrelated costs are expected to reach into the billions. Wall Street analysts at Sanford C. Bernstein \& Co. estimated the medical claims liability at $\$ 12$ billion associated with the acknowledgment by the manufacturer that use of the drug appeared to increase the risk of cardiovascular (CV) events. ${ }^{3}$ The Bernstein potential liability projection was based upon an estimated 49,000 persons who had taken $50 \mathrm{mg}$ of rofecoxib for any length of time or who took any dose of rofecoxib for more than 18 months. Even before the recall, more than 200 lawsuits had been filed by persons claiming to have been harmed by Vioxx. ${ }^{2}$ Whether these litigation estimates prove accurate will be determined by the courts over the course of time; however, the act of market withdrawal alone warrants the attention of clinicians and administrators making pharmaceutical treatment and purchasing decisions.

Costs and management issues relating to the appropriate and cost-effective use of cyclooxygenase- 2 selective (COX-2) inhibitors have been front-line subjects for managed care since the FDA approved the first COX-2 drug on December 31, 1998. No drug class has received more attention in articles in JMCP.4-9 With manufacturer sales of approximately $\$ 1.8$ billion in the United States in 2003 for rofecoxib and approximately $\$ 5.4$ billion for the class of COX-2 inhibitors, the expenditures for these drugs understandably have the attention of every health plan and drug plan sponsor in the United States. ${ }^{10}$

Clinicians and researchers will likely pore over the accumulated data from the published and unpublished clinical trials to affirm the wisdom of withdrawing rofecoxib from the market and to determine the magnitude of the $\mathrm{CV}$ risk posed by rofecoxib and the remaining COX-2 drugs-celecoxib and valdecoxib. In retrospect, however, we should not be too surprised by the findings that rofecoxib poses an increase in $\mathrm{CV}$ risk and that the marginal gastrointestinal (GI) protective benefits come at a high cost.

Looking back, rofecoxib is not the first "new" nonsteroidal anti-inflammatory drug (NSAID) to be withdrawn from the market. In the 1980s, benoxaprofen (Opren) was withdrawn from the market after photosensitivity, hepatotoxicity, and other adverse events appeared. ${ }^{11}$ Another NSAID, bromfenac (Duract), was withdrawn from the U.S. market in 1998 when its use was associated with hepatoxocity. ${ }^{12}$

After only 18 months on the U.S. market, clinical trial data on the adverse cardiac effects of rofecoxib began to appear in the medical literature. In November 2000, the results of the VIGOR (Vioxx Gastrointestinal Outcomes Research) ${ }^{13}$ study were published, reporting a $0.4 \%$ incidence of heart attack in rofecoxib users ( $50 \mathrm{mg}$ per day) versus $0.1 \%$ for naproxen users (1,000 mg per day), a rate 4 times higher for rofecoxib (absolute risk difference $=0.3 \%$, number needed to harm $=333$ ). The VIGOR study involved 8,076 patients who were at least 50 years of age (or at least 40 years of age and on long-term steroid therapy) and followed for an average of 9 months; concurrent aspirin use was not allowed in the VIGOR study.

Since publication of the results of the VIGOR study, evidence has continued to accumulate regarding the adverse effects of COX-2 drugs. In April 2004, the cardiac risk associated with the use of rofecoxib was reported in the results of a matched controlled study of 54,475 Medicare beneficiaries aged 65 years or older with a comprehensive drug benefit (the Pennsylvania Pharmaceutical Assistance Contract for the Elderly or the New Jersey Pharmaceutical Assistance Program for the Aged and Disabled) who received rofecoxib, celecoxib, or other nonselective NSAIDs during 1998-2000.14 Solomon et al. found a $24 \%$ increased risk of acute myocardial infarction (MI) in patients taking rofecoxib compared with those taking celecoxib for 90 days or less; however, there was no observed increased risk for rofecoxib compared with patients who did not take NSAIDs.

In June 2004, examination of population data over an 8.5-year period that ended in early 2002 found that the use of NSAIDs among Ontario's older population increased from $14.0 \%$ just before the introduction of COX-2 inhibitors to $19.8 \%$ by the end of the observation period $(P<0.01)$, representing an absolute increase of more than 90,000 additional persons annually using NSAIDs. ${ }^{15}$ The increase in use of all NSAIDs was attributed entirely to the use of COX-2 inhibitors rather than a switch from nonselective NSAIDs to COX-2 inhibitors. Before the introduction of COX-2 inhibitors, the rate of hospitalization for upper GI hemorrhage had been decreasing; after their introduction, it increased from 15.4 to 17.0 per 10,000 older persons (number needed to harm of $6,250, P<0.01$ ). This represents an absolute increase of more than 650 hospitalizations for upper GI hemorrhage annually. In other words, this population-based study found a $41 \%$ rise in overall NSAID use, due entirely to the use of COX-2 inhibitors, which was accompanied by a $10 \%$ increase in hospitalization rates for upper GI hemorrhage. Although 
ecological studies of this nature cannot prove that use of COX-2 inhibitors was directly responsible for the increased number of hospitalizations for upper GI bleeding, there appears to be an association between the increased use of COX-2 inhibitors and an increase in hospitalizations due to the large incremental increase in the use of these drugs.

The results of a study financed by the FDA were presented at a conference of epidemiologists in Bordeaux, France, on August 25, 2004. Evaluation of the medical records of 1.4 million members of Kaiser Permanente (Oakland, CA) found 8,199 heart attacks or sudden death between 1999 and $2001 .{ }^{16}$ Patients taking the highest recommended daily dosage of rofecoxib $(50 \mathrm{mg})$ had 3 times the risk of heart attack and sudden cardiac death as those not taking traditional nonselective NSAIDs. Rofecoxib users of either $12.5 \mathrm{mg}$ or $25 \mathrm{mg}$ per day also had a 50\% higher risk of heart attack compared with celecoxib patients.

When first confronted with the results of the VIGOR study, the manufacturer of rofecoxib suggested that the difference in the rates of MI was due to the cardioprotective effects of the comparator drug, naproxen, rather than a potential threat posed by rofecoxib. Noted cardiologist Eric J. Topol, MD, chairman of the Department of Cardiovascular Medicine at The Cleveland Clinic, referred to a press release by the manufacturer of rofecoxib on May 22, 2001 ("Merck Reconfirms Favorable Cardiovascular Safety of Vioxx"), as the first of a "relentless series of publications" including "numerous papers in the peer-reviewed medical literature by Merck employees and their consultants." ${ }^{17}$ In a serious warning letter, one of only a few such letters each year, the FDA sanctioned the manufacturer of rofecoxib for "selectively" presenting the hypothetical explanation that naproxen, the drug used in comparisons with rofecoxib in the VIGOR study, helps prevent CV events, thus making the rate of $\mathrm{CV}$ events with rofecoxib appear artificially inflated. The FDA said, "In fact, the situation is not at all clear," and that there are "no adequate and well-controlled studies" to support this assertion. The manufacturer was instructed by the FDA to develop a plan to immediately cease this line of promotional activities, which it deemed "misleading," and to send a "Dear Doctor" letter to all health care providers who were or may have been exposed to these promotions. The manufacturer responded to the FDA letter in a statement, "We continue to stand behind the overall and CV safety profile of Vioxx." ${ }^{18}$

Given the accumulation of evidence regarding the adverse effects of rofecoxib and the media attention that has accompanied it, perhaps now would be a good time to revisit the evidence for the entire class of nonselective NSAIDs and COX-2 drugs and to reassess the value equation for each of these classes of drugs.

Data from one of the newer COX-2 drugs, lumiracoxib (Prexige), a second-generation drug still in clinical trials, has demonstrated some GI benefits, but, as with the previous COX-2 drugs, are they worth the cost? The Therapeutic Arthritis
Research and Gastrointestinal Event Trial (TARGET) ${ }^{19}$ randomized 18,325 patients with osteoarthritis who were aged 50 years or older to treatment with lumiracoxib $400 \mathrm{mg}$ daily ( $n=9,156)$, naproxen $500 \mathrm{mg}$ twice daily $(n=4,754)$, or ibuprofen 800 mg thrice daily $(n=4,415)$ for 52 weeks. In the overall study population, $0.32 \%$ (29 of 9,117) of patients taking lumiracoxib experienced ulcer complications versus $0.91 \%$ (83 of 9,127 ) of patients taking nonselective NSAIDs (absolute risk reduction $=0.0059$, number needed to treat $=169$ ). It is important to note that $39 \%$ of the patients did not complete the study, and the reduction in ulcer complications was not significant in patients who were taking aspirin; for the patients taking aspirin, the hazard ratio was 0.79 (95\% confidence interval [CI], 0.40-1.55; $P=0.488$ ). In the patients not using aspirin, $0.20 \%$ of patients taking lumiracoxib experienced an ulcer complication versus $0.92 \%$ of patients taking NSAIDs (absolute risk reduction $=0.0072$, number needed to treat $=139$ ). In the overall population, $0.65 \%$ (59 of 9,117) of those taking lumiracoxib and $0.55 \%$ (50 of 9,127) of those taking NSAIDs reached the CV end point, defined as MI, stroke, or CV death (hazard ratio 1.14; $95 \% \mathrm{CI}, 0.78-1.66 ; P=0.507$ ). Therefore, you would have to treat 169 patients with lumiracoxib for 52 weeks to prevent 1 ulcer complication from occurring in patients treated with naproxen or ibuprofen. Assuming a drug cost of $\$ 3$ per day (a conservative estimate), it would cost a total of $\$ 185,055$ for lumiracoxib to prevent 1 ulcer complication in this population. A drug cost of $\$ 4$ per day would increase the direct cost of lumiracoxib to $\$ 246,740$ to prevent 1 ulcer complication. In addition, the excess number of MIs in lumiracoxib users compared with naproxen users $(0.38 \%$ versus $0.21 \%)$ is concerning. ${ }^{20}$

Although the increased rate of $\mathrm{CV}$ events in patients treated with lumiracoxib was not statistically significant, it does not mean that it is not real or clinically significant. The TARGET study excluded most patients with a history of MI, stroke, coronary artery bypass surgery, congestive heart failure or other significant coronary artery disease; it is thus difficult to draw conclusions regarding the true risk of $\mathrm{CV}$ events in a general population of users. More data are needed to adequately assess the $\mathrm{CV}$ risk of lumiracoxib use. In response to rising concerns about the CV safety profile of all COX-2 drugs, the manufacturer of lumiracoxib has delayed its filing and is apparently launching an investigation to assess its CV safety.

In a search of the English-language medical literature for articles published between January 1998 and February 2001 on the subject of selective COX-2 inhibitors, Mukherjee, Nissen, and Topol evaluated the data from 2 large randomized controlled trials (RCTs) and 2 smaller RCTs of 1,000 or more patients each. ${ }^{21}$ The VIGOR study, an RCT of 8,076 patients, found a relative risk of 2.38 for confirmed adjudicated thrombotic CV events (MI, unstable angina, cardiac thrombosis, resuscitated cardiac arrest, sudden or unexplained death, 
ischemic stroke, and transient ischemic attacks) with rofecoxib treatment (50 mg per day) versus naproxen (1,000 mg per day). The absolute incidence of adjudicated CV events was $1.14 \%$ for rofecoxib versus $0.50 \%$ for naproxen (number needed to harm $=$ 156). There were 111 patients $(2.74 \%$ of 4,047 rofecoxib patients) with CV events rated as "serious" by FDA medical review versus 50 patients in the naproxen group (1.24\% of 4,029 patients). The second large RCT was CLASS (Celecoxib Long-term Arthritis Safety Study)—a study of 8,059 patients treated mostly for osteoarthritis. The CLASS trial found no significant difference in CV events (MI, stroke, and death) between celecoxib (400 mg twice per day) and 2 NSAIDs (75 mg diclofenac twice daily or $800 \mathrm{mg}$ ibuprofen thrice daily); however, in contrast to the VIGOR study, aspirin use (325 mg per day or more) was permitted.

Mukherjee, Nissen, and Topol also found that the annualized MI rates for rofecoxib and celecoxib (in VIGOR and CLASS, respectively) were significantly higher compared with a group of 23,407 placebo patients aggregated in a meta-analysis from 4 aspirin prevention trials (U.S. Physicians' Health Study, the U.K. Doctors Study, the Thrombosis Prevention Trial, and the Hypertension Optimal Treatment): $0.74 \%$ annualized MI incidence for rofecoxib and $0.80 \%$ for celecoxib versus $0.52 \%$ for placebo, a relative difference of $42 \%$ for rofecoxib and $54 \%$ for celecoxib ( $P=0.04$ and $P=0.02$, for rofecoxib and celecoxib, respectively). Once again, this type of comparison cannot be used to conclude that a direct causal relationship exists between the use of COX-2 drugs and an increased incidence of MI. However, when added to all the other data that have been accumulating on the subject, it does suggest an association, which was ultimately borne out by the manufacturer's voluntary withdrawal of rofecoxib.

In addition to the 2 RCTs (CLASS and VIGOR), Mukherjee, Nissen, and Topol examined 2 studies of CV event rates submitted to the FDA—Study 085 and Study 090. ${ }^{21}$ Both studies were RCTs involving 1,042 patients (Study 085) and 978 patients (Study 090) taking $12.5 \mathrm{mg}$ per day of rofecoxib or 1,000 mg per day of nabumetone or placebo. There were $7 \mathrm{CV}$ events in the rofecoxib groups $(0.9 \%)$ versus $4 \mathrm{CV}$ events for nabumetone $(0.5 \%)$ and $1 \mathrm{CV}$ event for placebo $(0.25 \%)$. Lowdose aspirin for cardiac protection was allowed in both Study 085 and Study 090.

Collectively, these data provide substantial evidence that long-term use of rofecoxib, and perhaps other COX-2 drugs, increases the risk of $\mathrm{CV}$ events when compared with traditional nonselective NSAIDs, and possibly placebo.

Given these findings and the fact that COX-2 drugs are no more effective at treating arthritis pain than traditional nonselective NSAIDs, it is time to rethink the use of this class of drugs. Although COX-2 inhibitors reduce ulcer complications compared with traditional NSAIDs, the absolute risk reduction of GI events is small. In fact, neither celecoxib or valdecoxib have been able to earn FDA approval for labeling regarding GI protective effects compared with nonselective NSAIDs; thus, only 1 COX-2 inhibitor (rofecoxib) has received FDA approval for this label, and it is has been withdrawn from the market because of CV safety concerns.

In addition to CV safety concerns and the small absolute benefit in reducing upper GI bleeding complications, one has to consider the high costs of COX-2 inhibitors compared with traditional nonselective NSAIDs, many of which are available in generic form and over-the-counter. Spiegel et al. performed a cost-effectiveness analysis evaluating 2 strategies for managing a hypothetical cohort of 60 year-old-patients with osteoarthritis or rheumatoid arthritis who were not taking aspirin and required long-term NSAID therapy for moderate-tosevere pain. ${ }^{22}$ One strategy involved treatment with rofecoxib $25 \mathrm{mg}$ daily or celecoxib $200 \mathrm{mg}$ daily while the other involved use of a nonselective NSAID at the maximum FDA-approved dose (modeled after naproxen at $500 \mathrm{mg}$ twice daily). They found that use of COX-2 drugs versus nonselective NSAIDs cost an incremental $\$ 275,809$ to gain 1 additional qualityadjusted life-year (QALY). For comparative purposes, this is more than twice the cost per QALY of initiating dialysis in patients with renal failure. When they factored in the potential for CV events, the incremental cost increased to $\$ 395,324$ per QALY gained. This is more than 3.5 times the cost per QALY associated with initiating intensive care and mechanical ventilation in patients with respiratory failure. For patients with a history of bleeding ulcer, the incremental cost per QALY decreased to $\$ 55,803$. They concluded that the reduction in GI event rates was not offset by the increased costs of COX-2 inhibitors, that they may be cost effective in patients with a history of bleeding ulcers, and that the costs of COX-2 inhibitors would have to be reduced by $90 \%$ for them to become dominant.

When one factors in the high costs of COX-2 drugs, the small absolute reduction in GI complications, and the increased risk of $\mathrm{CV}$ events, it is difficult to justify the use of COX-2 drugs as first-line therapy for the management of arthritis or other musculoskeletal pain, except perhaps in patients at high risk for upper GI bleeding complications and at low risk for CV events. In the meantime, patients taking other COX-2 inhibitors, and trials involving current and new COX-2 inhibitors, should be closely monitored to assess the incidence of $\mathrm{CV}$ events and ensure that patients are not being unduly harmed.

Eduardo Ortiz, MD, MPH Associate Chief of Staff and Attending Physician Washington DC Veterans Affairs Medical Center 50 Irving St., NW Washington, DC 20422 eortiz@med.va.gov 


\section{Editorial}

\section{DISCLOSURES}

The author discloses no potential bias or conflict of interest relating to this article. He is solely responsible for the content of this article; no statements or views in this article should be construed as official endorsements or positions of the Department of Veterans Affairs or the federal government.

\section{REFERENCES}

1. Merck Withdraws Arthritis Drug Vioxx. Washington Post. Available at: http://www.washingtonpost.com/wp-dyn/articles/A61885-2004Sep30.html. Accessed September 30, 2004

2. Merck Withdraws Arthritis Medication: Vioxx Maker Cites Users' Health Risks. Washington Post. Available at: http://www.washingtonpost.com/wpdyn/articles/A63157-2004Sep30.html. Accessed October 1, 2004.

3. Evans RT, Gandhi D, Kranson DB. MRK: What's the maximum number of legitimate Vioxx claimants? Bernstein Research Call. October 14, 2004

4. Curtiss FR. Step-therapy edits for PPIs and COX-2 drugs-what we do and do not know. J Manag Care Pharm. 2004;10(4):356.

5. Cox ER, Henderson R, Motheral BR. Health plan member experience with point-of-service prescription step therapy. J Manag Care Pharm. 2004;10(4): 291-98.

6. Stacy J, Shaw E, Arledge MD, Howell-Smith D. Pharmacoeconomic modeling of prior-authorization intervention for COX-2 specific inhibitors in a 3-tier copay plan. J Manag Care Pharm. 2003;9(4):327-34.

7. Bull SA, Conel C, Campen DH. Relationship of clinical factors to the use of COX-2 selective NSAIDs within an arthritis population in a large HMO. J Manag Care Pharm. 2003;8(4):252-58

8. Curtiss FR. Cost-effective use of COX-2 drugs and NSAIDs. J Manag Care Pharm. 2002:8(4):295-96.

9. Tucker G, Moore A, Avant D, Monteiro M. A cost analysis of four benefit strategies for managing a COX-2 inhibitor. J Manag Care Pharm. 2001;7(3): 224-27.

10. Martinez B, Wilde Mathews A, Lublin JS, Winslow R. Merck pulls Vioxx from market after link to heart problems. Wall Street Journal. October 1 , 2004:Al, Al2.
11. Smith R. Medical journals and pharmaceutical companies: uneasy bedfellows. BMJ. 2003;326:1202-05.

12. Sharpe R. Painkiller-how a drug approved by the FDA turned into a lethal failure. Wall Street Journal. September 30, 1998:A1.

13. Bombardier C, Laine L, Reicin A, et al. Comparison of upper gastrointestinal toxicity of rofecoxib and naproxen in patients with rheumatoid arthritis. VIGOR Study Group. N Engl J Med. 2000;343:1520-28.

14. Solomon DH, Schneeweiss S, Glynn RJ, et al. Relationship between selective cyclooxygenase- 2 inhibitors and acute myocardial infarction in older adults. Circulation. 2004;109(17):2068-73. [April 19, 2004 (epub ahead of print.)

15. Mamdani M, Juurlink DN, Kopp A, et al. Gastrointestinal bleeding after the introduction of COX-2 inhibitors: ecological study. BMJ. 2004:328:141516

16. Anonymous. Study finds Vioxx increases heart attack risk. Reuters. August 25, 2004.

17. Topol EJ. Failing the public health—rofecoxib, Merck and the FDA. New Engl J Med. 2004;351:1707-09.

18. Adams C. FDA warns Merck for Vioxx promotions. Wall Street Journal. September 25, 2001:B5.

19. Schnitzer TJ, Burmester GR, Mysler E, et al. Comparison of lumiracoxib with naproxen and ibuprofen in the Therapeutic Arthritis Research and Gastrointestinal Event Trial (TARGET), reduction in ulcer complications: randomized controlled trial. Lancet. 2004;364:665-74

20. Topol EJ, Falk GW. A coxib a day won't keep the doctor away. Lancet. 2004:364(9435):639-40

21. Mukherjee D, Nissen SE, Topol EJ. Risk of cardiovascular events associated with selective COX-2 inhibitors. JAMA. 2001 286(8):954-59.

22. Spiegel BMR, Targownik L, Dulai GS, Gralnek IM. The cost-effectiveness of cyclooxygenase-2 selective inhibitors in the management of chronic arthritis Ann Intern Med. 2003;138(10):795-806. 